TRANSACTIONS OF THE

AMERICAN MATHEMATICAL SOCIETY

Volume 354, Number 10, Pages 3869-3882

S 0002-9947(02)03053-2

Article electronically published on June 4, 2002

\title{
HOCHSCHILD HOMOLOGY CRITERIA FOR TRIVIAL ALGEBRA STRUCTURES
}

\author{
MICHELINE VIGUÉ-POIRRIER
}

\begin{abstract}
We prove two similar results by quite different methods. The first one deals with augmented artinian algebras over a field: we characterize the trivial algebra structure on the augmentation ideal in terms of the maximality of the dimensions of the Hochschild homology (or cyclic homology) groups. For the second result, let $X$ be a 1-connected finite CW-complex. We characterize the trivial algebra structure on the cohomology algebra of $X$ with coefficients in a fixed field in terms of the maximality of the Betti numbers of the free loop space.
\end{abstract}

\section{INTRODUCTION}

Let $A$ be a unital algebra over a field $k$. By definition, the Hochschild homology of $A$ with coefficients in $A$ (called here Hochschild homology) is the homology of the Hochschild complex $\left(C_{*}(A), b\right)$ (see section 1) and is denoted $H H_{*}(A)=$ $\bigoplus_{n \geq 0} H H_{n}(A)$, Lo]. If $A$ is a finite dimensional $k$-vector space, then each homology group is finite dimensional and the $n$-th Betti number of $H H_{*}(A)$ is defined as the dimension of the $k$-vector space $H H_{n}(A)$.

In $\S 1$, we focus our attention on augmented algebras $A=k \oplus \bar{A}$ where $\bar{A}$ is the augmentation ideal and we assume that $\bar{A}$ is a finite dimensional $k$-vector space. When $\bar{A} \cdot \bar{A}=0$, the calculation of the Betti numbers of $H H_{*}(A)$ has been carried out by many people, [L-Q], [Ro], [Pa]. If $A$ is any augmented algebra with augmentation ideal $\bar{A}$, we can consider $A_{t}=k \oplus \bar{A}_{t}$ the augmented algebra with augmentation ideal $\bar{A}_{t}$, where $\bar{A}_{t}=\bar{A}$ as a $k$-vector space and $\bar{A}_{t} \cdot \bar{A}_{t}=0$. We first show (theorem 1.3) that

(1) $\operatorname{dim} H H_{n}(A) \leq \operatorname{dim} H H_{n}\left(A_{t}\right)$ for $n \geq 0$,

(2) $\operatorname{dim} H C_{n}(A) \leq \operatorname{dim} H C_{n}\left(A_{t}\right)$ for $n \geq 0$

where $\left(H C_{n}(\cdot)\right)_{n \geq 0}$ are the cyclic homology groups ([Lo and definition below).

Then (theorems 1.4, 1.6) we prove that the inequalities above become equalities if and only if the multiplication is trivial on the augmentation ideal, so that we characterize the trivial algebra structure on $A$ in terms of the maximality of the Betti numbers of the Hochschild homology or cyclic homology groups.

Received by the editors March 23, 2001 and, in revised form, March 15, 2002.

2000 Mathematics Subject Classification. Primary 13N05, 18F25, 55P62.

Key words and phrases. Augmented algebra, Hochschild homology, cyclic homology, free loop space, minimal model of a differential graded algebra. 
More precisely, we first show that if (2) is an equality for some $n \geq 1$ and $\bar{A}$ is a nilpotent ideal, then $A=A_{t}$ as algebras. After that, we show that if (1) is an equality for some $n \geq 1$ and $A$ is a commutative algebra, then $A=A_{t}$ as algebras.

More generally, if $(A, d)$ is a differential graded algebra over a field $k$, we can define Hochschild and cyclic homology groups as above $\mathrm{Go},[\mathrm{Lo}$.

Let $X$ be a simply connected space. A result of Burghelea-Fiedorowicz $[\mathrm{B}-\mathrm{F}$, or Goodwillie [Go], or Jones [Jo] asserts that

$$
\begin{aligned}
& H_{*}\left(X^{S^{1}}, k\right)=H H_{*}\left(C_{*}(\Omega X, k)\right), \\
& H_{*}^{S^{1}}\left(X^{S^{1}}, k\right)=H C_{*}\left(C_{*}(\Omega X), k\right)
\end{aligned}
$$

as $k$-graded vector spaces, where $C_{*}(\Omega X, k)$ is the differential graded algebra of singular chains on the Moore loop space of $X$, and $X^{S^{1}}$ is the free loop space on $X$, i.e., the space of all continuous maps from the circle into $X$. The group $S^{1}$ acts on $X^{S^{1}}$ by rotating the loops, and we denote by $H_{*}^{S^{1}}\left(X^{S^{1}}, k\right)$ the homology of the Borel construction associated to this action.

The study of the Betti numbers of the free loop space is motivated by its applications to geometry. A famous example is the Gromoll-Meyer theorem [G-M] which asserts the following: if $X$ is a 1-connected compact manifold such that the sequence of Betti numbers dim $H^{n}\left(X^{S^{1}}, k\right)$ is not bounded, then there exist infinitely many closed distinct geodesics on $X$ for any Riemannian metric. Roos Ro and Parhizgar $[\mathrm{Pa}]$ compute explicitly these Betti numbers when $X$ is a wedge of spheres of the same dimension; in this case, $H^{*}(X, k)$ is an augmented algebra and the multiplication is trivial on $\tilde{H}=\bigoplus_{n>0} H^{n}(X, k)$.

In $\S 2$, we give, in the context of topology, similar results to those of $\S 1$. The proofs are quite different and rely on the notion of an Adams-Hilton model for a space [A-H]. Let $H=\bigoplus_{n \geq 0} H_{n}$ be a graded $k$-vector space with $H_{0}=k, H_{1}=0$ and $\bar{H}=\bigoplus_{n>0} H_{n}$ finite dimensional. We consider the wedge of spheres $X_{t}=\bigvee_{1 \leq i \leq r} S^{n_{i}}$, where the sequence $\left(n_{1}, \ldots, n_{r}\right)$ corresponds to a basis of the vector space $\bar{H}$. We have $H^{*}\left(X_{t}, k\right)=\operatorname{Hom}(H, k)$.

Theorem 2.1 asserts that if $X$ is any finite complex with $H_{*}(X, k)=H$, then the Betti numbers of the free loop space on $X$ and the equivariant free loop space on $X$ are bounded by those of $X_{t}$. Conversely, we prove (theorem 2.2 and theorem $2.4)$ that if the Betti numbers are the same, then the multiplication is trivial on $\tilde{H}^{*}(X, k)=\bigoplus_{n>0} H^{n}(X, k)$

As in the algebraic case, we characterize the trivial algebra structure on $H^{*}(X, k)$ in terms of the maximality of the Betti numbers of the free loop space.

Recall from [Vi1] that if $X_{t}=\bigvee_{1 \leq i \leq r} S^{n_{i}}, r \geq 2$, we have proved that the sequence of Betti numbers of the free loop space grows exponentially. When $X$ is a hyperbolic space, many attempts have been made to prove the exponential growth of the Betti numbers, La1, La2. Results in this paper convince us that the proof will be harder when multiplication is not trivial on $\tilde{H}^{*}(X, k)$. 


\section{The Hochschild And CYClic HOMOlogy of AUGMENTED Algebras}

In the following, we always consider augmented algebras over a fixed field $k$. We recall, from [Lo], the definitions of Hochschild and cyclic homology.

The Hochschild homology groups $\left(H H_{n}(A)\right)_{n \geq 0}$ are the homology groups of the Hochschild complex $\left(C_{*}(A), b\right)$. If $\bar{A}$ is the augmentation ideal of $A$, we define $C_{*}(A)=\bigoplus_{n \geq 0} C_{n}(A)$ and $b$ by the following formulas:

$$
\begin{gathered}
C_{n}(A)=A \otimes \bar{A}^{\otimes n}, \\
b\left(a_{0} \otimes a_{1} \otimes \ldots \otimes a_{n}\right) \\
=\sum_{i=0}^{n-1}(-1)^{i} a_{0} \otimes \ldots \otimes a_{i} a_{i+1} \otimes \ldots \otimes a_{n}+(-1)^{n} a_{n} a_{0} \otimes a_{1} \otimes \ldots \otimes a_{n-1}
\end{gathered}
$$

where $a_{0} \in A, a_{i} \in \bar{A}$ if $i \geq 1$.

All the tensor products are over $k$.

We introduce the cyclic permutation $t_{n}: \bar{A}^{\otimes n} \rightarrow \bar{A}^{\otimes n}$ defined by $t_{n}\left(a_{1} \otimes \ldots \otimes\right.$ $\left.a_{n}\right)=(-1)^{n-1} a_{n} \otimes a_{1} \otimes \ldots \otimes a_{n-1}$.

From Loday, [L], Proposition 2.2.14, the reduced cyclic homology groups $H \tilde{C}_{n}(A):=H C_{n}(A) / H C_{n}(k)$ can be computed as the homology groups of the complex $\bar{A}^{\otimes(n+1)} /\left(I d-t_{n+1}\right)$ endowed with the differential induced by $b$, when char $k=0$, or char $k=p$ and $n<p-1$.

Proposition $1.1([\mathrm{Ro}])$. Let $A$ be an augmented algebra, where the augmentation ideal $\bar{A}$ is finite dimensional and satisfies $\bar{A} \cdot \bar{A}=0$. Then

1. $H H_{n}(A)=\operatorname{Coker}\left(\mathrm{Id}-t_{n+1}\right) \oplus \operatorname{Ker}\left(\mathrm{Id}-t_{n}\right)$ as $k$-vector spaces for all $n>0$;

2. if char $k=0, H \tilde{C}_{n}(A)=\operatorname{Coker}\left(\mathrm{Id}-t_{n+1}\right)$ for all $n>0$.

Corollary 1.2. Let $A$ be an augmented algebra, where the augmentation ideal $\bar{A}$ is finite dimensional and satisfies $\bar{A} \neq 0, \bar{A} \cdot \bar{A}=0$. Then

1. $H H_{n}(A) \neq 0$ for all $n>0$;

2. if char $k=0, H \tilde{C}_{2 n}(A) \neq 0$ for all $n \geq 0$ and $H C_{2 n+1}(A) \neq 0$ for all $n \geq 0$ if $\operatorname{dim} \bar{A} \geq 2$.

Proof. Let $x$ be a nonzero element in $A$. Then the element $x \otimes x \otimes \ldots \otimes x$ belongs to $\bar{A}^{\otimes(2 n+1)} \cap \operatorname{Ker}\left(\mathrm{Id}-t_{2 n+1}\right)$.

If $\operatorname{dim} \bar{A} \geq 2$, let $x$ and $y$ be two independent elements in the $k$-vector space $\bar{A}$.

Put $X=\sum_{i=1}^{2 n} X_{i}$ with $X_{i}=(-1)^{i-1} x \otimes \ldots \otimes y \otimes \ldots \otimes x$ with $y$ at the $i$ th place in the tensor product $\bar{A}^{\otimes(2 n)}$; we check that $X$ belongs to $\bar{A}^{\otimes(2 n)} \cap \operatorname{Ker}\left(\mathrm{Id}-t_{2 n}\right)$ so that $H C_{2 n-1}(A) \neq 0$.

Remark. If char $k=p, p>0$, part 2 of proposition 1.1 and corollary 1.2 remain valid for $n<p-1$.

Theorem 1.3. Let $A$ be an augmented algebra over a field $k$. Let $\bar{A}$ be its augmentation ideal, with $\operatorname{dim}_{k} \bar{A}$ finite. Let $A_{t}=k \oplus \bar{A}_{t}$ be the augmented algebra with trivial multiplication on $\bar{A}_{t}$ and $\bar{A}=\bar{A}_{t}$ as a $k$-vector space. We assume that char $k=0$, or char $k \neq 0$ and there exists $N \geq 2$ such that $\bar{A}^{N}=0$ in $A$; then we have 
1. $\operatorname{dim} H H_{n}(A) \leq \operatorname{dim} H H_{n}\left(A_{t}\right)$ for all $n \geq 0$;

2. $\operatorname{dim} H C_{n}(A) \leq \operatorname{dim} H C_{n}\left(A_{t}\right)$ for all $n \geq 0$.

Remark. If $A$ is local, or if $A=\bigoplus_{n \geq 0} A_{n}$ is a graded algebra with $A_{0}=k$, there exists $N \geq 2$ such that $\bar{A}^{N}=0$. Otherwise, it cannot be true; for example, if $A=k[X] /\left(X^{2}-X\right)$, we have $\bar{A}^{N}=\bar{A}=(X)$ for all $N \geq 1$. In this case, parts 1$)$ and 2) of the proposition remain true for $n<\operatorname{char} k-1$.

Proof. If char $k=0$, the reduced cyclic homology can be computed using the reduced Connes complex $\bar{C}_{*}^{\lambda}=\bigoplus_{n} \bar{C}_{n}^{\lambda}$ with $\bar{C}_{n}^{\lambda}=\bar{A}^{\otimes n+1} /\left(I d-t_{n+1}\right)$. We denote by $\bar{b}$ its differential; we have seen that $H \tilde{C}_{n}\left(A_{t}\right)=\bar{A}^{\otimes n+1} /\left(I d-t_{n+1}\right)$, and we have $H \tilde{C}_{n}(A)=\left(\bar{A}^{\otimes n+1} /\left(I d-t_{n+1}\right), \bar{b}\right)$. This implies trivially that $\operatorname{dim} H \tilde{C}_{n}(A) \leq$ $\operatorname{dim} H \tilde{C}_{n}\left(A_{t}\right)$. Using the Connes long exact sequence, we see that

$$
\operatorname{dim} H H_{n}(A) \leq \operatorname{dim} H \tilde{C}_{n-1}(A)+\operatorname{dim} H \tilde{C}_{n}(A) \text { for } n \geq 1 ;
$$

so we have $\operatorname{dim} H H_{n}(A) \leq \operatorname{dim} H \tilde{C}_{n-1}\left(A_{t}\right)+\operatorname{dim} H \tilde{C}_{n}\left(A_{t}\right)$.

$A_{t}$ can be considered as a graded algebra with $A_{0}=k$; so we can use Goodwillie's trick ([ $[\mathrm{Lo}]$, theorem 4.1.13) and we have

$$
\operatorname{dim} H H_{n}\left(A_{t}\right)=\operatorname{dim} H \tilde{C}_{n-1}\left(A_{t}\right)+\operatorname{dim} H \tilde{C}_{n}\left(A_{t}\right) .
$$

This gives us $\operatorname{dim} H H_{n}(A) \leq \operatorname{dim} H H_{n}\left(A_{t}\right)$ for all $n$.

If $k$ is an arbitrary field, we use a spectral sequence argument to prove theorem 1.3. We define an increasing filtration on the Hochschild complex as follows:

$$
\begin{array}{lll}
\mathcal{F}_{-p}\left(C_{n}(A)\right)=A \otimes\left(\bar{A}^{p}\right)^{\otimes n} & \text { for } & p \geq 1, \\
\mathcal{F}_{k}\left(C_{n}(A)\right)=C_{n}(A) & \text { for } & k \geq 0 .
\end{array}
$$

With the additional hypothesis that there exists $N \geq 2$ such that $\bar{A}^{N}=0$, this filtration is bounded and gives rise to a spectral sequence $\left(E_{* *}^{r}, d^{r}\right)$ converging to $H H_{*}(A)$ with

$$
\begin{aligned}
& E_{-p, n+p}^{0}=A \otimes\left(\frac{\bar{A}^{p}}{\bar{A}^{p+1}}\right)^{\otimes n} \quad \text { for } \quad p \geq 1, \\
& E_{k q}^{0}=0 \quad \text { if } \quad k \geq 0 .
\end{aligned}
$$

We check that $d^{0}\left(\left(\lambda+\bar{a}_{0}\right) \otimes \bar{a}_{1} \otimes \ldots \otimes \bar{a}_{n}\right)=\lambda\left(I d-t_{n}\right)\left(\bar{a}_{1} \otimes \ldots \otimes \bar{a}_{n}\right)$ where $\bar{a}_{0} \in \bar{A}$ and $\bar{a}_{i} \in \bar{A}^{p} / \bar{A}^{p+1}$ for $i \geq 1$.

So we have $\bigoplus_{p} E_{-p, n+p}^{1}=H H_{n}\left(A_{t}\right)$. This implies

$$
\operatorname{dim} H H_{n}(A) \leq \operatorname{dim} H H_{n}\left(A_{t}\right) .
$$

To prove 2), we use the reduced bicomplex $\bar{B}(A)$ to compute cyclic homology ([Lo], page 58), and we define on it an increasing filtration as above.

Now, we are interested in algebras $A$ for which inequality 1 or 2 of theorem 1.3 becomes an equality.

Theorem 1.4. Let $A$ be an augmented algebra over a characteristic zero field $k$. Let $\bar{A}$ be its augmentation ideal. We assume that $\bar{A}$ is a finite dimensional $k$-vector space and there exists $N \geq 2$ such that $\bar{A}^{N}=0$, and $\bar{A} \neq 0$. Let $A_{t}=k \oplus \bar{A}_{t}$ be 
the augmented algebra with trivial multiplication on $\bar{A}_{t}$, and $\bar{A}_{t}=\bar{A}$ as a k-vector space. Suppose that there exists $n \geq 1$ such that

$$
\operatorname{dim} H C_{n}(A)=\operatorname{dim} H C_{n}\left(A_{t}\right) .
$$

Then the multiplication is trivial in the augmented algebra $A$ (namely, $A$ is isomorphic to $A_{t}$, as augmented algebras).

Proof. We have $H \tilde{C}_{n}(A)=H_{n}\left(\bar{A}^{\otimes(n+1)} /\left(I d-t_{n+1}\right), \bar{b}\right) . \quad$ If $\operatorname{dim} H C_{n}(A)=$ $\operatorname{dim} H C_{n}\left(A_{t}\right)$, then $\operatorname{dim} H \tilde{C}_{n}(A)=\operatorname{dim} H \tilde{C}_{n}\left(A_{t}\right)$. This is equivalent to the facts that $\bar{b}=0$ is zero on $\bar{A}^{\otimes(n+1)} /\left(I d-t_{n+1}\right)$ and $\bar{b}$ is zero on $\bar{A}^{\otimes(n+1)} /\left(I d-t_{n+2}\right)$. This implies that there exists $m \geq 1$ such that

$$
b\left(\bar{A}^{\otimes(2 m+1)}\right) \quad \text { belongs to } \quad\left(I d-t_{2 m}\right)\left(\bar{A}^{\otimes(2 m)}\right) .
$$

But we have

$$
\left(I d-t_{2 m}\right)\left(\bar{A}^{\otimes 2 m}\right)=\operatorname{Ker}\left(I d+t_{2 m} \ldots+t_{2 m}^{2 m-1}\right) .
$$

We can consider $x \otimes \ldots \otimes x$ in $\bar{A}^{\otimes(2 m+1)}$ where $x$ is a nonzero element in $\bar{A}$.

We have $b(x \otimes \ldots \otimes x)=2 x^{2} \ldots \otimes x+\sum_{i=2}^{2 m}(-1)^{i-1} x \otimes \ldots \otimes x^{2} \ldots \otimes x$.

We check that $X=\left(I d+t_{2 m}+\ldots+t_{2 m}^{2 m-1}\right) \circ b(x \otimes \ldots \otimes x)=\sum_{1 \leq i \leq 2 m} X_{i}$ with $X_{i}=(-1)^{i-1}(2 m+1) x \otimes \ldots \otimes x^{2} \otimes \ldots \otimes x$ and $x^{2}$ at the $i$ th place.

Let $\left(x, e_{2}, \ldots, e_{d}\right)$ be a basis of the $k$-vector space $\bar{A}$. We can write $x^{2}=\lambda x+$ $\sum_{2 \leq i \leq d} \lambda_{i} e_{i}$ with $\lambda \in k, \lambda_{i} \in k, 2 \leq i \leq d$.

The condition $X=0$ implies that $(2 m+1) \lambda_{i}=0$ for $2 \leq i \leq d$. Since $2 m+1<p$, we should have $\lambda_{i}=0$ for $2 \leq i \leq d$. The hypothesis $\overline{\bar{A}}^{N}=0$ implies $\lambda=0$, so that $x^{2}=0$, for any $x \in \bar{A}$.

If $\operatorname{dim} \bar{A}=1$, we have proved that $\bar{A} \cdot \bar{A}=0$.

If $\operatorname{dim} \bar{A} \geq 2$, consider two independent elements $x$ and $y$ in $\bar{A}$; we have $0=$ $(x+y)^{2}=x^{2}+y^{2}+x y+y x$, and so we have

$$
x y+y x=0 .
$$

From the hypothesis, we have seen that there exists $m \geq 1$ such that $b\left(\bar{A}^{\otimes 2 m}\right)$ belongs to $\left(I d-t_{2 m-1}\right)\left(\bar{A}^{\otimes(2 m-1)}\right)$.

Let $X=y \otimes x \otimes \ldots \otimes x$ in $\bar{A}^{\otimes 2 m}$,

$$
b(X)=(y x-x y) \otimes x \ldots \otimes x-y \otimes x^{2} \otimes x \ldots \otimes x \ldots+y \otimes x \ldots \otimes x^{2} .
$$

Since $x^{2}=0$ and $x y=-y x$, we have

$$
b(x)=2 y x \otimes x \otimes x \otimes \ldots \otimes x .
$$

Let $\left(x, y, e_{3}, \ldots, e_{d}\right)$ be a basis of the $k$-vector space $\bar{A}$. We can write $y x=\mu x+$ $\nu y+\sum_{i \geq 3} \alpha_{i} e_{i}$ with $\mu, \nu, \alpha_{i} \in k$.

If $b(X)$ belongs to $\left(I d-t_{2 m-1}\right)\left(\bar{A}^{\otimes(2 m-1)}\right)=\operatorname{Ker}\left(I d+t_{2 m-1}+\ldots+t_{2 m-1}^{2 m-2}\right)$, then we should have $(2 m-1) \mu=0,2 \nu=0,2 \alpha_{i}=0$. This proves that $\bar{A} \cdot \bar{A}=0$.

So, $A$ is an augmented commutative algebra isomorphic to $A_{t}$.

Remark. Theorem 4.1 remains valid if char $k=p, p>0$, and $n<p-2$. 
Example 1.5. Let $A=k[X] /\left(X^{2}-X\right)$ and $A_{t}=k[X] / X^{2}$. We check, [B-V], that

$$
\begin{aligned}
& H \tilde{C}_{2 n}\left(A_{t}\right)=H \tilde{C}_{2 n}(A)=k, \\
& H C_{2 n+1}\left(A_{t}\right)=H C_{2 n+1}(A)=0, \\
& H H_{n}\left(A_{t}\right)=k \text { for all } n>0, \\
& H H_{n}(A)=0 \text { for all } n>0 .
\end{aligned}
$$

This shows that the hypothesis $\bar{A}^{N}=0$ cannot be omitted in theorem 1.4. On the other hand, the Hochschild homology groups of $A_{t}$ and $A$ are quite distinct.

This observation leads us to state the following for commutative algebras.

Theorem 1.6. Let $A$ be an augmented commutative algebra over a characteristic zero field $k$. Let $\bar{A}$ be its augmentation ideal and assume that $\bar{A}$ has finite dimension. Let $A_{t}=k \oplus \bar{A}_{t}$ be the augmented algebra with trivial multiplication on $\bar{A}_{t}$ and $\bar{A}_{t}=\bar{A}$ as a k-vector space. Suppose that there exists $n \geq 1$ such that

$$
H H_{n}(A)=H H_{n}\left(A_{t}\right) .
$$

Then the multiplication is trivial in the augmented algebra $A$ (namely $A$ is isomorphic to $A_{t}$ as augmented algebras).

Proof. Assume that there exists $n \geq 1$ such that

$$
H H_{n}(A)=H H_{n}\left(A_{t}\right) \text {. }
$$

From the Connes exact sequence, we see that

$$
\operatorname{dim} H H_{n}(A) \leq \operatorname{dim} H \tilde{C}_{n}(A)+\operatorname{dim} H \tilde{C}_{n-1}(A) .
$$

From theorem 1.3, we know that

$$
\operatorname{dim} H \tilde{C}_{n-1}(A)+\operatorname{dim} H \tilde{C}_{n}(A) \leq \operatorname{dim} H \tilde{C}_{n-1}\left(A_{t}\right)+\operatorname{dim} H \tilde{C}_{n}\left(A_{t}\right) .
$$

But, if $\operatorname{dim} H H_{n}(A)=\operatorname{dim} H H_{n}\left(A_{t}\right)$ for some $n$, we have

$\operatorname{dim} H H_{n}\left(A_{t}\right)=\operatorname{dim} H \tilde{C}_{n}\left(A_{t}\right)+\operatorname{dim} H \tilde{C}_{n-1}\left(A_{t}\right) \leq \operatorname{dim} H \tilde{C}_{n}(A)+\operatorname{dim} H \tilde{C}_{n-1}(A)$.

The two inequalities imply that

$$
\begin{aligned}
& \operatorname{dim} H \tilde{C}_{n}(A)=\operatorname{dim} H \tilde{C}_{n}\left(A_{t}\right), \\
& \operatorname{dim} H \tilde{C}_{n-1}(A)=\operatorname{dim} H \tilde{C}_{n-1}\left(A_{t}\right) .
\end{aligned}
$$

The first part of the proof of theorem 1.4 implies that, for any nonzero element $x$ in $\bar{A}$, there exists $\lambda \in k$, such that $x^{2}=\lambda x$.

Denote by $\left(x_{1}, \ldots, x_{m}\right)$ a $k$-basis of $\bar{A}$; so there exist $\lambda_{i} \in k$ for $1 \leq i \leq m$ such that $x_{i}^{2}=\lambda_{i} x_{i}$. If $m=1$, we recover example 1.5 and the theorem is true. If $m \geq 2$, consider two independent elements $x_{i}, x_{j}, i \neq j$ in $\bar{A}$. Using the fact that there exist $(\mu, \nu) \in k^{2}$ such that

$$
\begin{aligned}
& \left(x_{i}+x_{j}\right)^{2}=\mu\left(x_{i}+x_{j}\right), \\
& \left(x_{i}-x_{j}\right)^{2}=\nu\left(x_{i}-x_{j}\right),
\end{aligned}
$$

we show that

$$
x_{i} x_{j}=\frac{\lambda_{j}}{2} x_{i}+\frac{\lambda_{i}}{2} x_{j} .
$$


This proves that $A=S / I$ where $S$ is the polynomial algebra $k\left[X_{1}, \ldots, X_{m}\right]$ and $I$ is generated by

$$
f_{i}=X_{i}^{2}-\lambda_{i} X_{i}, 1 \leq i \leq m, \quad g_{i j}=X_{i} X_{j}-\frac{\lambda_{j}}{2} X_{i}-\frac{\lambda_{i}}{2} X_{j}, 1 \leq i<j \leq m .
$$

Using the Jacobian criterion, it is easy to check that if there exists $i$ with $\lambda_{i} \neq 0$, then $A$ is smooth over $k$. Since $A$ is artinian, the module of Kahler differential forms $\Omega_{A / k}^{1}$ should be zero.

Using the Hochschild-Kostant-Rosenberg theorem [H-K-R, we see that all the Hochschild homology groups $H H_{n}(A)$ should be zero for $n \geq 1$.

We have seen, in corollary 1.2, that $H H_{n}\left(A_{t}\right)$ is nonzero for all $n$.

So, if there exists $n \geq 1$ such that $H H_{n}(A)=H H_{n}\left(A_{t}\right)$, then $H H_{n}(A) \neq 0$ and $A$ is not smooth over $k$. This occurs only if $\lambda_{i}=0$ for all $i \in\{1, \ldots, m\}$. In that case $A=k\left[X_{1}, \ldots, X_{m}\right] /\left(\left(X_{i}^{2}\right)_{1 \leq i \leq m},\left(X_{i} X_{j}\right)_{1 \leq i<j \leq m}\right)$.

Remark. Theorem 1.6 remains valid if char $k=p>3$ and $1 \leq n<p-1$.

\section{Characterization of SPaCes with given homology vector SPace in} TERMS OF THE BETTI NUMBERS OF THE FREE LOOP SPACE

In the following, we fix an arbitrary field $k$.

Let $X$ be a simply-connected finite $C W$-complex. Denote by $X^{S^{1}}$ the space of all continuous maps from the circle into $X$. Since the circle acts on $X^{S^{1}}$ by rotating loops, we can apply to $X^{S^{1}}$ the Borel construction and we get a space denoted by $E S^{1} \times_{S^{1}} X^{S^{1}}$ whose homology, denoted $H_{*}^{S^{1}}\left(X^{S^{1}}, k\right)$, is called the equivariant homology of the free loop space.

The starting point for our study is a result of Burghelea-Fiedorowicz $\mathrm{B}-\mathrm{F}$, Goodwillie [Go], and J. Jones [Jo, which asserts the following:

$$
\begin{aligned}
& H_{*}\left(X^{S^{1}}, k\right)=H H_{*}\left(C_{*}(\Omega X, k)\right), \\
& H_{*}^{S^{1}}\left(X^{S^{1}}, k\right)=H C_{*}\left(C_{*}(\Omega X, k)\right)
\end{aligned}
$$

where $C_{*}(\Omega X, k)$ is the differential graded algebra of singular chains on the Moore loop space of $X$.

The definitions of Hochschild and cyclic homology can be extended easily to the category of differential graded chain algebras ( $[\mathrm{Lo}$, chapter 5).

Results of $\S 1$ lead us to ask the following question, in the topological framework:

Let $H=\bigoplus_{n \geq 0} H_{n}$ be a finite dimensional graded vector space over an arbitrary field $k$, with $H_{0}=k, H_{1}=0, \bar{H}=\bigoplus_{n \geq 1} H_{n} \neq 0$. Let $\left(e_{1}, \ldots, e_{r}\right)$ be a homogeneous basis of $\bar{H}$. Then we put $X_{t}=\bigvee_{1 \leq i \leq r} S^{n_{i}}$, where $n_{i}$ is the degree of $e_{i}$. Hence $H_{*}\left(X_{t}, k\right)$ is canonically isomorphic to $H$. Now, if $X$ is any finite $C W$-complex with $H_{*}(X, k)=H$, can we compare the Betti numbers of the free loop space on $X$ and on $X_{t}$, and characterize spaces $X$ such that $X^{S^{1}}$ and $X_{t}^{S^{1}}$ have the same Betti numbers?

Unfortunately, this problem cannot be tackled as in the algebraic context, because the notion of Connes complex, defined as a quotient of the Hochschild complex, cannot not be extended to differential graded algebras. 
The main tool is the Adams-Hilton model for a $C W$-complex whose 1-skeleton is trivial, $\mathrm{A}-\mathrm{H}$. To such a space $X$, they associate a differential graded chain algebra $A_{X}$ and a morphism

$$
\theta: A_{X} \longrightarrow C_{*}(\Omega X, k),
$$

which induces an isomorphism in homology.

From [B-L], there exists a free differential graded algebra $(T(V), d)$ and a morphism $\theta_{0}:(T(V), d) \rightarrow A_{X}$, which induces an isomorphism in homology. Furthermore:

1. $V=\bigoplus_{n \geq 1} V_{n}, \quad V_{n}=H_{n+1}(X, k)$,

2. $d=d_{2}+d_{3}+\ldots+d_{n}+\ldots$ with $d_{n}(V) \subset V^{\otimes n}$ (we say that $(T(V), d)$ is minimal),

3. $d_{2}$ is dual to the multiplication on $H^{*}(X, k)$.

It is proved in $[\mathrm{E}-\mathrm{H}]$ that $d_{2} V \subset \mathbb{L}^{2}(V)$ where $\mathbb{L}(V)$ is the free Lie algebra generated by $V$.

A classical result of Goodwillie Go asserts that

$$
\begin{aligned}
& H H_{*}\left(C_{*}(\Omega X)\right)=H H_{*}(T(V), d), \\
& H C_{*}\left(C_{*}(\Omega X)\right)=H C_{*}(T(V), d)
\end{aligned}
$$

as graded $k$-vector spaces.

Now, we give a similar result to theorem 1.3.

Theorem 2.1. Let $H=\bigoplus_{n \geq 0} H_{n}$ be a finite dimensional graded $k$-vector space with $H_{0}=k, H_{1}=0$. Let $X$ be any finite complex with $H_{*}(X, k)=H$ and denote by $X_{t}$ the wedge of spheres such that $H_{*}\left(X_{t}, k\right)=H$. Then we have

1. $\operatorname{dim} H_{n}\left(X^{S^{1}}, k\right) \leq \operatorname{dim} H_{n}\left(X_{t}^{S^{1}}, k\right)$,

2. $\operatorname{dim} H_{n}^{S^{1}}\left(X^{S^{1}}, k\right) \leq \operatorname{dim} H_{n}^{S^{1}}\left(X_{t}^{S^{1}}, k\right)$

for all $n \geq 0$.

Proof. In [Vi2], we exhibit in theorem 1.5 (resp. theorem 2.4) a short complex that computes $H H_{*}(T(V), d)$ (resp. $H C_{*}(T(V), d)$ ). We use them here.

Recall that $H H_{*}(T(V), d)=H_{*}(T(V) \oplus T(V) \otimes \bar{V}, \delta)$ where

$$
\begin{aligned}
& \bar{V}=s V, \quad(s V)_{n}=V_{n-1}, \\
& \delta_{\mid T(V)}=d
\end{aligned}
$$

if $a \in T(V), v \in \bar{V}, \delta(a \otimes \bar{v})=(-1)^{|a|+|\bar{v}|}(I d-\tau)(a \otimes \bar{v})+\tilde{d}(a \otimes \bar{v})$.

$I: T(V) \otimes \bar{V} \rightarrow T(V)$ satisfies $I(a \otimes \bar{v})=a v$, $\tau: T(V) \otimes \bar{V} \rightarrow T(V)$ satisfies $\tau(a \otimes \bar{v})=(-1)^{|a| \cdot|v|} v a$,

$\tilde{d}: T(V) \otimes \bar{V} \rightarrow T(V) \otimes \bar{V}$ satisfies $\tilde{d}(a \otimes \bar{v})=d a \otimes \bar{v}+(-1)^{|a|} S(a, d v)$, $S: T(V) \otimes T^{+}(V) \rightarrow T(V) \otimes \bar{V}$ satisfies

$$
S\left(a \otimes v_{1} \ldots v_{p}\right)=\sum_{i=1}^{p} \pm v_{i+1} \ldots v_{p} a v_{1} \ldots v_{i-1} \otimes \bar{v}_{i},
$$

if $a \in A, v_{i} \in V$, and $|a|$ means the degree of $a$. 
We define a bounded filtration on $\left(C_{*}, \delta\right)=(T(V) \oplus T(V) \otimes \bar{V}, \delta)$ by

$$
\begin{aligned}
& \mathcal{F}_{p}\left(C_{n}\right)=C_{n} \quad \text { if } \quad p \geq n, \\
& \mathcal{F}_{n-1}\left(C_{n}\right)=(T(V) \otimes \bar{V})_{n}, \\
& \mathcal{F}_{p}\left(C_{n}\right)=0 \quad \text { if } \quad p \leq n-2 .
\end{aligned}
$$

This determines a convergent spectral sequence $E_{p q}^{r}$ with

$$
\begin{aligned}
& E_{p q}^{r}=0 \quad \text { if } \quad q \neq 0 \quad \text { or } \quad q \neq 1, \\
& E_{p o}^{1}=[T(V) / \operatorname{Im}(I-\tau)]_{p}, \\
& E_{p-1,1}=[\operatorname{Ker}(I-\tau)]_{p} .
\end{aligned}
$$

So we have, for a fixed $p \geq 0$,

$$
E_{p o}^{1} \oplus E_{p-1,1}^{1}=H H_{p}(T(V), 0)=H_{p}\left(X_{t}^{S^{1}}, k\right) .
$$

Since $E_{p q}^{r}$ converges to $H H_{*}(T(V), d)=H_{*}\left(X^{S^{1}}, k\right)$, part (1) is proved.

To prove part (2), we should recall the definition of the short complex $\left(\mathcal{B}_{*}, D\right)$ given in [Vi2] to compute $H C_{*}(T(V), d)$ :

$$
\begin{aligned}
& \mathcal{B}_{*}=k[u] \otimes C_{*} \quad \text { with } \quad|u|=2, \\
& D=0 \quad \text { on } \quad k[u], \\
& D=\delta=d \quad \text { on } \quad T(V), \\
& D\left(u^{n} \otimes a \otimes \bar{v}\right)=u^{n} \otimes \delta(a \otimes \bar{v}) \quad \text { if } \quad a \in T(V), \bar{v} \in \bar{V}, n \in \mathbb{N}, \\
& D\left(u^{n} \otimes v_{1} \ldots v_{m}\right)=u^{n} \otimes d\left(v_{1} \ldots v_{m}\right)+u^{n-1} \otimes S\left(1, v_{1} \ldots v_{m}\right)
\end{aligned}
$$

if $v_{i} \in V, 1 \leq i \leq m$ and $n \geq 1$.

In fact, we will consider the reduced complex $\tilde{\mathcal{B}}_{*}=\mathcal{B}_{*} / k[u]$ with the induced differential $\tilde{D}$, that computes reduced cyclic homology

$$
H \tilde{C}_{*}(T(V), d):=H C_{*}(T(V), d) / H C_{*}(k) .
$$

We define a bounded filtration on $(\tilde{\mathcal{B}}, \tilde{D})$ as follows:

$$
\begin{aligned}
& \mathcal{F}_{p}\left(\tilde{\mathcal{B}}_{n}\right)=\tilde{\mathcal{B}}_{n} \quad \text { for } \quad p \geq n \\
& \mathcal{F}_{n-2 q}\left(\tilde{\mathcal{B}}_{n}\right)=\bigoplus_{i \geq q}\left(T^{+}(V) \otimes u^{i}\right)_{n} \oplus\left(\bigoplus_{j \geq q} T(V) \otimes \bar{V} \otimes u^{j}\right)_{n} \quad \text { for } \quad q>0, \\
& \mathcal{F}_{n-2 q-1}\left(\tilde{\mathcal{B}}_{n}\right)=\bigoplus_{i \geq q+1}\left(T^{+}(V) \otimes u^{i}\right)_{n} \oplus\left(\bigoplus_{j \geq q} T(V) \otimes \bar{V} \otimes u^{j}\right)_{n} \quad \text { for } \quad q \geq 0 .
\end{aligned}
$$

The induced spectral sequence $\left(E_{p q}^{r}, d^{r}\right)$ satisfies

$$
\begin{aligned}
& E_{n-2 q, 2 q}^{0}=\left(T^{+}(V) \otimes u^{q}\right)_{n} \quad \text { for } \quad q \geq 0, \\
& E_{n-2 q-1,2 q+1}^{0}=\left(T(V) \otimes \bar{V} \otimes u^{q}\right)_{n} \quad \text { for } \quad q \geq 0 .
\end{aligned}
$$

The differential $d^{0}$ is precisely the differential which computes $H \tilde{C}_{*}(T(V), 0)$ using the complex of theorem 2.4 of [Vi2] with zero differential on $T(V)$. So we have

$$
\bigoplus_{p+q=n} E_{p q}^{1}=H \tilde{C}_{n}(T(V), 0)=\tilde{H}_{n}^{S^{1}}\left(X_{t}^{S^{1}}, k\right) .
$$

Since $E_{p q}^{r}$ converges to $H \tilde{C}_{*}(T(V), d)=\tilde{H}_{*}^{S^{1}}\left(X^{S^{1}}, k\right)$, part $(2)$ of theorem 2.1 is proved. 
Example. Considering $X=\mathbb{C} P^{2}$, we have $H^{*}(X, k)=k \oplus k x_{2} \oplus k x_{2}^{2}$ with $\left|x_{2}\right|=2$. We show, in [Vi2], that $\operatorname{dim} H_{n}\left(X^{S^{1}}\right)=1$ for all $n \geq 1$. The corresponding space $X_{t}$ is $S^{2} \bigvee S^{4}$ and we have shown in Vi1] that the sequence $\operatorname{dim} H_{n}\left(X_{t}^{S^{1}}\right)$ has exponential growth.

Now we are interested in spaces $X$ for which inequality (1) or (2) of theorem 2.1 becomes an equality.

Theorem 2.2. Let $H=\bigoplus_{n \geq 0} H_{n}$ be a finite dimensional graded $k$-vector space with $H_{0}=k, H_{1}=0$ and $N=\sup \left\{n \mid H_{n} \neq 0\right\}$ strictly positive. Let $X_{t}$ be the wedge of spheres such that $H_{*}\left(X_{t}, k\right)=H$. Let $X$ be a finite complex such that $H_{*}(X, k)=$ $H$. We assume that either char $k=0$ or $\operatorname{char} k=p \geq 3 N-3$, and

$$
\operatorname{dim} H_{n}^{S^{1}}\left(X^{S^{1}}, k\right)=\operatorname{dim} H_{n}^{S^{1}}\left(X_{t}^{S^{1}}, k\right) \text { for all } n \leq 3 N-3 .
$$

Then the multiplication is trivial on $\tilde{H}^{*}(X, k)=\bigoplus_{n>0} H^{*}(X, k)$.

Corollary 2.3. With the same hypothesis as in theorem 2.2, we assume furthermore that there exists an integer $r \geq 1$ such that $H_{n}=0$ for $n \leq r$ and $n \geq 3 r+2$. Then we have

$$
H_{*}(\Omega X, k)=H_{*}\left(\Omega X_{t}, k\right)=T\left(s^{-1} \bar{H}\right)
$$

as graded algebras, where $\bar{H}=\bigoplus_{n>0} H_{n}$ and $\left(s^{-1} \bar{H}\right)_{n}=H_{n+1}$.

Theorem 2.4. Let $H=\bigoplus_{n>0} H_{n}$ be a finite dimensional graded $k$-vector space with $H_{0}=k, H_{1}=0$, and $N=\sup \left\{n \mid H_{n} \neq 0\right\}$ nonzero. Let $X_{t}$ be the wedge of spheres such that $H_{*}\left(X_{t}, k\right)=H$. Let $X$ be a finite $C W$-complex with $H_{*}(X, k)=H$. We assume that either char $k=0$, or char $k=p>3 N-3$, and

$$
\operatorname{dim} H_{n}\left(X^{S^{1}}, k\right)=\operatorname{dim} H_{n}\left(X_{t}^{S^{1}}, k\right) \text { for all } n \leq 3 N-3 .
$$

Then the multiplication is trivial on $\tilde{H}^{*}(X, k)=\bigoplus_{n>0} H^{*}(X, k)$.

Corollary 2.5. With the same hypothesis as in theorem 2.4, we assume furthermore that there exists an integer $r \geq 1$ such that $H_{n}=0$ for $n \leq r$ and $n \geq 3 r+2$. Then we have

$$
H_{*}(\Omega X, k)=H_{*}\left(\Omega X_{t}, k\right)=T\left(s^{-1} \bar{H}\right)
$$

as graded $k$-algebras.

As in the algebraic context, the proof of theorem 2.4 is easy from theorem 2.2. We leave it to the reader.

Proof of theorem 2.2. From theorem 2.5 of [Vi2], we have

$$
\begin{aligned}
\tilde{H}_{n}^{S^{1}}\left(X_{t}^{S^{1}}, k\right)=H \tilde{C}_{n}((T(V), 0))= & \bigoplus \bigoplus_{q \geq 0} \bigoplus_{m \geq 1} H_{2 q, n-2 q}\left(\mathbb{Z} / m \mathbb{Z}, V^{\otimes m}\right) \\
& \oplus\left(\bigoplus_{q \geq 0} \bigoplus_{m \geq 1} H_{2 q+1, n-2 q-1}\left(\mathbb{Z} / m \mathbb{Z}, V^{\otimes m}\right)\right) .
\end{aligned}
$$


This is exactly the term $\bigoplus_{p+q=n} E_{p q}^{1}$ in the spectral sequence defined on the complex $\left(\mathcal{B}_{*}, D\right)$ in the proof of theorem 2.1. If we assume that $\operatorname{dim} H_{n}^{S^{1}}\left(X_{t}^{S^{1}}, k\right)=$ $\operatorname{dim} H_{n}^{S^{1}}\left(X^{S^{1}}, k\right)$, then the differentials $d_{r}$ on $\bigoplus_{p+q=n} E_{p q}^{r}$ should be zero, for all $r \geq 1$.

If we assume that char $k=0$, or $n \leq$ char $k$ if char $k \neq 0$, then all the groups $H_{q, n-q}\left(\mathbb{Z} / m \mathbb{Z}, V^{\otimes m}\right)$ are zero if $q \neq 0$. So we have

$$
H \tilde{C}_{n}(T(V), 0)=E_{n, 0}^{1}=\bigoplus_{m \geq 1}\left[\frac{V^{\otimes m}}{\operatorname{Im}\left(I-\tau_{m}\right)}\right]_{n}
$$

and the differential $d^{1}: E_{n, 0}^{1} \rightarrow E_{n-1,0}^{1}$ is induced by the differential $d$ of $T(V)$.

Assume now that $H_{n}^{S^{1}}\left(X_{t}^{S^{1}}, k\right)=H_{n}^{S^{1}}(X, k)$ for all $n \leq 3 N-3$ (with the additional hypothesis $3 N-3 \leq \operatorname{char} k$ if $\operatorname{char} k \neq 0)$.

Then we have necessarily

$$
d\left(V^{\otimes m}\right)_{n} \subset \bigoplus_{m \geq 2}\left[\operatorname{Im}\left(I-\tau_{m}\right)\right]_{n-1}
$$

for all $m \geq 1$, for all $n \leq 3 N-3$ if char $k=0$, or for all $n \leq 3 N-3 \leq$ char $k$ if char $k \neq 0$.

We can write $d=d_{2}+d_{3}+\ldots+d_{i}+\ldots$ with $d_{i}(V) \subset V^{\otimes i} ; i \geq 2$.

We will prove that $d_{2}=0$.

Notice that if char $k=3$, then the hypothesis of the theorem implies that $N=2$; so we have $V=V_{1}$ and $d=0$. In the following, we assume that char $k>3$. Suppose there exist elements $v \in V$ such that $d_{2} v \neq 0$. Let $v$ be one of them of lowest degree $p$ for this property.

From $[\mathrm{E}-\mathrm{H}]$, we can write

$$
d_{2} v=\sum_{i<j} \lambda_{i j}\left(u_{i} \otimes u_{j}-(-1)^{\left|u_{i}\right| \times\left|u_{j}\right|} u_{j} \otimes u_{i}\right)+\sum_{i} \mu_{i} u_{i} \otimes u_{i}
$$

with $\lambda_{i j} \in k, \mu_{i} \in k$, and $\left\{u_{i}\right\}$ is a $k$-basis of $\bigoplus_{n<p} V_{n}$.

If $p$ is even, then $\mu_{i}=0$, for all $i$.

If $p$ is odd, consider a term $\mu_{i} u_{i} \otimes u_{i}$ that appears in $d_{2} v$; we have $v \otimes u_{i} \in\left(V^{\otimes 2}\right)_{n}$ with $n \leq 3 N-3$, so that $d_{2}\left(v \otimes u_{i}\right)$ should belong to $\operatorname{Im}\left(I-\tau_{3}\right)_{n-1}=\operatorname{Ker}\left(I+\tau_{3}+\right.$ $\left.\tau_{3}^{2}\right)_{n-1}$ since char $k>3$.

We have

$$
\begin{aligned}
& d_{2}\left(v \otimes u_{i}\right)=d_{2}(v) \otimes u_{i}=\sum_{k<j} \lambda_{k j}\left(u_{k} \otimes u_{j} \otimes u_{i}-(-1)^{\left|u_{i}\right| \times\left|u_{j}\right|}\left(u_{j} \otimes u_{k} \otimes u_{i}\right)\right. \\
&+\sum_{j \neq i} \mu_{j} u_{j} \otimes u_{j} \otimes u_{i}+\mu_{i} u_{i} \otimes u_{i} \otimes u_{i} \\
&\left(I+\tau_{3}+\tau_{3}^{2}\right)\left(d_{2}\left(v \otimes u_{i}\right)\right)=3 \mu_{i} u_{i} \otimes u_{i} \otimes u_{i}+X
\end{aligned}
$$

where $X$ is a sum of monomials of $V^{\otimes 3}$, each of them containing some $u_{j}$ with $j \neq i$.

This implies $\mu_{i}=0$ for all $i$.

So we have $d_{2} v=\sum_{i<j} \lambda_{i j}\left(u_{i} \otimes u_{j}-(-1)^{\left|u_{i}\right| \times\left|u_{j}\right|} u_{j} \otimes u_{i}\right)$. 
If $p$ is even, then $p-1$ is odd, so that necessarily, $\left|u_{i}\right|$ or $\left|u_{j}\right|$ is odd. Assume that $\left|u_{i}\right|$ is odd and consider

$$
\left(I+\tau_{3}+\tau_{3}^{2}\right)\left(d_{2}\left(v \otimes u_{i}\right)\right)=-2 \lambda_{i j}\left[u_{i} \otimes u_{i} \otimes u_{j}+u_{j} \otimes u_{i} \otimes u_{i}+u_{i} \otimes u_{j} \otimes u_{i}\right]+X
$$

where $X$ belongs to $V^{\otimes 3}$, and $X$ is a sum of monomials, all containing some $u_{k}$, $k \neq i, k \neq j$.

Since $\left(I+\tau_{3}+\tau_{3}^{2}\right)\left(d_{2}\left(v \otimes u_{i}\right)\right)$ should be zero, we have $\lambda_{i j}=0$. This proves that $d_{2} v=0$ if $|v|$ is even.

If $p$ is odd, we fix $i$ and $j, i<j$ and we consider $v \otimes u_{i} \otimes u_{j}$ that belongs to $\left(V^{\otimes 3}\right)_{n}$ with $n \leq 3 N-3$, so that $d_{2}\left(v \otimes u_{i} \otimes u_{j}\right)=\left(d_{2} v\right) \otimes u_{i} \otimes u_{j}$ should belong to $\operatorname{Im}\left(I-\tau_{4}\right)$, or equivalently to $\operatorname{Ker}\left(I+\tau_{4}+\tau_{4}^{2}+\tau_{4}^{3}\right)=K$ since char $k=0$, or char $k>3$.

We can suppose that $i=1, j=2$, so that we write

$$
\begin{gathered}
d_{2}\left(v \otimes u_{1} \otimes u_{2}\right)=\lambda_{12}\left(u_{1} \otimes u_{2} \otimes u_{1} \otimes u_{2}-(-1)^{\left|u_{1}\right| \times\left|u_{2}\right|} u_{2} \otimes u_{1} \otimes u_{1} \otimes u_{2}\right) \\
\quad+\sum_{\substack{i<j \\
(i, j \neq(1,2)}} \lambda_{i j}\left(u_{i} \otimes u_{j} \otimes u_{1} \otimes u_{2}-(-1)^{\left|u_{j}\right| \times\left|u_{i}\right|} u_{j} \otimes u_{i} \otimes u_{1} \otimes u_{2}\right) .
\end{gathered}
$$

If $d_{2}\left(v \otimes u_{1} \otimes u_{2}\right)$ belongs to $K$, then we should have that

$$
\lambda_{12}\left(u_{1} \otimes u_{2} \otimes u_{1} \otimes u_{2}-(-1)^{\left|u_{1}\right| \times\left|u_{2}\right|} u_{2} \otimes u_{1} \otimes u_{1} \otimes u_{2}\right)
$$

belongs to $K$.

This implies $\left(1+(-1)^{\left|u_{1}\right|+\left|u_{2}\right|}\right) \lambda_{12}=0$, but $\left|u_{1}\right|+\left|u_{2}\right|=p-1$ is even, so that we should have $\lambda_{12}=0$. We have proved that

$$
d_{2} v=0 \quad \text { if }|v| \text { is odd. }
$$

Since $d_{2}$ is dual to the multiplication on $\tilde{H}^{*}(X, k)=\bigoplus_{n>0} H^{n}(X, k)$, $\mathrm{B}-\mathrm{L}$, we have immediately that the multiplication is trivial on $\tilde{H}^{*}(X, k)$.

Proofs of corollaries 2.3 and 2.5. It is proved in [An] that spaces $X$ satisfying the additional hypothesis of the corollaries are $k$-formal; and for such spaces, the differential $d$ of their Adams-Hilton model is just equal to $d_{2}$. Since we have proved that the hypotheses of theorems 2.2 or 2.4 imply that $d_{2}=0$, in fact, for these spaces, $d=0$ in the Adams-Hilton model; so there is a morphism of differential graded algebras $\theta:\left(T\left(s^{-1} \bar{H}\right), 0\right) \rightarrow C_{*}(\Omega X, k)$ that induces an isomorphism in homology; so we have

$$
H_{*}(\Omega X, k)=T\left(s^{-1} H_{*}(X)\right) .
$$

Remark. More generally, corollary 2.5 means that if a space $X$ is $k$-formal and satisfies the hypothesis of theorem 2.4, then $H_{*}(\Omega X, k)=T\left(s^{-1} H_{*}(X)\right)$ as algebras. If $k$ is the field of rational numbers, then $X$ has the same rational homotopy type as the wedge of spheres $X_{t}$. So, we can characterize wedge of spheres among other formal spaces with given homology as those having the largest Betti numbers for the free loop space.

If char $k$ is positive, the situation is more complicated, since there exist formal spaces $X$ with $H_{*}(\Omega X, k)=T\left(s^{-1} \bar{X}_{*}(X)\right)$, and the L.S. category of $X$ is two.

Remark. Consider the fibration $\Omega X \rightarrow X^{I} \stackrel{p}{\longrightarrow} X \times X$ where $X^{I}=\{f:[0,1] \rightarrow X\}$ and $p(f)=(f(0), f(1))$. Let $\Delta$ be the diagonal map $X \rightarrow X \times X$ defined by 
$\Delta(x)=(x, x)$. Then the free loop space $X^{S^{1}}$ is the total space of the pull-back of the fibration $p$. This gives rise to an Eilenberg-Moore spectral sequence in the second quadrant

$$
E_{2}^{-p q}=\operatorname{Tor}_{p}^{H^{*}(X \times X)}\left(H^{*}(X, k), H^{*}(X, k)\right)^{q} \Longrightarrow H^{*}\left(X^{S^{1}}, k\right) .
$$

If $X_{f}$ is a $k$-formal space with $H^{*}\left(X_{f}, k\right)=H^{*}(X, k)$ as graded algebras, the term $E_{2}$ is just $H^{*}\left(X_{f}^{S^{1}}, k\right)$; this implies that $\operatorname{dim} H^{n}\left(X^{S^{1}}, k\right) \leq \operatorname{dim} H^{n}\left(X_{f}^{S^{1}}, k\right)$, for all $n \geq 0$. We do not know examples of nonformal spaces $X$ such that $\operatorname{dim} H^{n}\left(X^{S^{1}}, k\right)=\operatorname{dim} H^{n}\left(X_{f}^{S^{1}}, k\right)$. In any case, it is easy to exhibit nonformal spaces $X$ such that

$$
\operatorname{dim} H^{n}(\Omega X, \mathbb{Q})=\operatorname{dim} H^{n}\left(\Omega X_{f}, \mathbb{Q}\right) \text { for all } n \geq 0 .
$$

It is sufficient to perturb the differential in the bigraded model of $H^{*}(X, \mathbb{Q})$ by adding a decomposable part $[\mathrm{H}-\mathrm{S}]$. So we conjecture that there exist nonformal spaces $X$ such that the Betti numbers of $X^{S^{1}}$ and $X_{f}^{S^{1}}$ are the same, and there is little hope to improve the conclusion of theorem 2.4.

\section{REFERENCES}

[An] D. Anick, A model of Adams Hilton type for fibre squares, Illinois J. Math., 29, (1985), 463-502. MR 86h:55009

[A-H] J. F. Adams and P. J. Hilton, On the chain algebra of a loop space, Comment. Math. Helv., 30, (1956), 305-330. MR 17:1119b

[B-L] H. Baues and J. M. Lemaire, Minimal models in homotopy theory, Math. Ann., 225, (1977), 219-242. MR 55:4174

[B-F] D. Burghelea and Z. Fiedorowicz, Cyclic homology and algebraic K-theory of spaces, Topology, 25, (1986), 303-317. MR 88i:18009b

[B-V] D. Burghelea and M. Vigué-Poirrier, Cyclic homology of commutative algebras, Lecture Notes in Mathematics, 1318, (1988), 51-72. MR 89k:18027

[E-H] El Haouari, P-Formalité des espaces, J. Pure Appl. Algebra, 78, (1992), 27-47. MR 93d:55016

[Go] T. Goodwillie, Cyclic homology, derivations and the free loop space, Topology, 24, (1985), 187-215. MR 87c:18009

[G-M] D. Gromoll and W. Meyer, Periodic geodesics on compact Riemannian manifolds, J. Differential Geom., 3, (1969), 493-510. MR 41:9143

[Jo] J. Jones, Cyclic homology and equivariant homology, Invent. Math., 87, (1987), 403-423. MR 88f: 18016

[H-S] S. Halperin and J. Stasheff, Obstructions to homotopy equivalences, Advances in Math., 32, (1979), 233-279. MR 80j:55016

$[\mathrm{H}-\mathrm{V}] \quad$ S. Halperin and M. Vigué-Poirrier, The homology of a free loop space, Pacific J. Math., 147, (1991), 311-324. MR 92e:55012

[H-K-R] G. Hochschild, B. Kostant, and A. Rosenberg, Differential forms on regular affine algebras, Trans. Amer. Math. Soc., 102, (1962), 383-408. MR 26:167

[La1] P. Lambrechts, Analytic properties of Poincaré series of spaces, Topology, 37, (1998), 1363-1370. MR 99e:55011

[La2] P. Lambrechts, On the Betti numbers of the free loop space of a coformal space, J. Pure Appl. Algebra, 161, (2001), 177-192. MR 2002d:55015

[Lo] J. L. Loday, Cyclic homology, Springer-Verlag, Berlin, (1992). MR 94a:19004

[L-Q] J. L. Loday and D. Quillen, Cyclic homology and the Lie algebra homology of matrices, Comment. Math. Helv., 59, (1984), 565-591. MR 86i:17003

[Pa] M. Parhizgar, On the cohomology ring of the free loop space of a wedge of spheres, Math. Scand, 80, (1997), 195-248. MR 98i:55014

[Ro] J. E. Roos, Homology of free loop spaces, cyclic homology, and nonrational PoincaréBetti series in commutative algebra, Lecture Note in Math., 1352, (1988), 173-189. MR 90f: 55020 
[Vi1] M. Vigué-Poirrier, Homotopie rationnelle et croissance du nombre de géodésiques fermées, Ann. Sci. Ecole Norm. Sup., 17, (1984), 413-431. MR 86h:58027

[Vi2] M. Vigué-Poirrier, Homologie de Hochschild et homologie cyclique des algèbres différentielles graduées, Astérisque 191, (1990), 255-267. MR 92e:19003

Université Paris-Nord, Institut Galilée, Département de Mathématiques, F-93430 Villetaneuse, France

E-mail address: vigue@math.univ-paris13.fr 